\title{
1 Glacier-fed stream biofilms harbour diverse 2 resistomes and biosynthetic gene clusters
}

3

4 Susheel Bhanu Busi ${ }^{1, \#, *}$, Laura de Nies ${ }^{1, \#}$, Paraskevi Pramateftaki², Massimo Bourquin²,

5 Leïla Ezzat², Tyler J. Kohler², Stilianos Fodelianakis², Grégoire Michoud², Hannes Peter²,

6 Michail Styllas², Matteo Tolosano², Vincent De Staercke ${ }^{2}$, Martina Schön², Valentina

$7 \quad$ Galata $^{1}$, Paul Wilmes ${ }^{1, *}$ and Tom Battin ${ }^{2}$

8

9 1Systems Ecology Group, Luxembourg Centre for Systems Biomedicine, University of

10 Luxembourg, Esch-sur-Alzette, Luxembourg

11 2Stream Biofilm \& Ecosystem Research Lab, ENAC, Ecole Polytechnique Fédérale de

12 Lausanne, Lausanne, Switzerland

13

$14 \quad$ \#Contributed equally to this work

15

16

17 Prof. Paul Wilmes (paul.wilmes@uni.lu)

18 Susheel Bhanu Busi (susheel.busi@uni.lu)

19

20

Running title: Resistome and biosynthetic gene clusters of glacier-fed streams

${ }^{*}$ Corresponding author(s):

21

22 Keywords: glacier-fed streams, metagenomics, antimicrobial resistance, biosynthetic gene

23 clusters, cross-domain interactions 


\section{Abstract}

\section{Background}

26 Antimicrobial resistance (AMR) is a universal phenomenon whose origins lay in natural

27 ecological interactions such as competition within niches, within and between micro- to

28 higher-order organisms. However, the ecological and evolutionary processes shaping

29 AMR need to be better understood in view of better antimicrobial stewardship. Resolving

30 antibiotic biosynthetic pathways, including biosynthetic gene clusters (BGCs), and

31 corresponding antimicrobial resistance genes (ARGs) may therefore help in

32 understanding the inherent mechanisms. However, to study these phenomena, it is

33 crucial to examine the origins of AMR in pristine environments with limited anthropogenic

34 influences. In this context, epilithic biofilms residing in glacier-fed streams (GFSs) are an

35 excellent model system to study diverse, intra- and inter-domain, ecological crosstalk.

\section{$37 \quad$ Results}

38 We assessed the resistomes of epilithic biofilms from GFSs across the Southern Alps

39 (New Zealand) and the Caucasus (Russia) and observed that both bacteria and

40 eukaryotes encoded twenty-nine distinct AMR categories. Of these, beta-lactam,

41 aminoglycoside, and multidrug resistance were both abundant and taxonomically

42 distributed in most of the bacterial and eukaryotic phyla. AMR-encoding phyla included

43 Bacteroidota and Proteobacteria among the bacteria, alongside Ochrophyta (algae)

44 among the eukaryotes. Additionally, BGCs involved in the production of antibacterial

45 compounds were identified across all phyla in the epilithic biofilms. Furthermore, we found

46 that several bacterial genera (Flavobacterium, Polaromonas, etc.) including

47 representatives of the superphylum Patescibacteria encode both ARGs and BGCs within 
48 close proximity of each other, thereby demonstrating their capacity to simultaneously

49 influence and compete within the microbial community.

50

51 Conclusions

52 Our findings highlight the presence and abundance of AMR in epilithic biofilms within

53 GFSs. Additionally, we identify their role in the complex intra- and inter-domain

54 competition and the underlying mechanisms influencing microbial survival in GFS epilithic

55 biofilms. We demonstrate that eukaryotes may serve as AMR reservoirs owing to their

56 potential for encoding ARGs. We also find that the taxonomic affiliation of the AMR and

57 the BGCs are congruent. Importantly, our findings allow for understanding how naturally

58 occurring BGCs and AMR contribute to the epilithic biofilms mode of life in GFSs.

59 Importantly, these observations may be generalizable and potentially extended to other

60 environments which may be more or less impacted by human activity. 
62 Background

63 Today, antimicrobial resistance (AMR) has become a well-known threat to human health

64 with an estimated number of 700,000 people per year dying of drug-resistant infections

65 [1]. The dramatic rise of antimicrobial resistance over the past decade has even led to the

66 moniker, "silent pandemic" [2]. Therefore, AMR is often directly associated with human

67 impacted environments with a global increase in resistant bacteria linked to the over- and

68 mis-use of antibiotics [3]. However, contrary to public perception, AMR is a natural

69 phenomenon, which has existed for billions of years [4]. Long before the rather recent

70 use of antibiotics in the clinical setting, microorganisms have used these, along with

71 corresponding protective mechanisms, to establish competitive advantages over other

72 microbes contending for the same environment and/or resources [5].

74 Microbes, in general, produce a range of secondary metabolites with diverse chemical

75 structures which in turn confer a variety of functions, including antibiotics [6]. Such

76 secondary metabolites including metal transporters and quorum sensing molecules $[7,8]$

77 are not directly associated with the growth of microorganisms themselves but instead are

78 known to provide benefits by acting as growth inhibitors against competing bacteria.

79 Consequently, many of these natural products have found their uses in industrial settings

80 as well as in human medicine as anti-infective drugs $[7,9,10]$. The biosynthetic pathways

81 responsible for producing these specialized metabolites are encoded by locally clustered

82 groups of genes known as 'biosynthetic gene clusters' (BGCs). Typically, BGCs include

83 genes for expression control, self-resistance, and metabolite export [11]. They can,

84 however, be further divided into various classes including non-ribosomal peptide

85 synthetases (NRPSs), type I and type II polyketide synthases (PKSs), terpenes, and 
86 bacteriocins alongside others [10]. NRPSs and PKSs specifically have been of interest

87 due to their known synthesis of putative antibiotics [12,13]. Furthermore, evidence

88 suggests that within these BGCs at least one resistance gene conferring resistance can

89 be found as a self-defense mechanism against the potentially harmful secondary

90 metabolites encoded by the BGC [14]. For instance, the tylosin-biosynthetic gene cluster

91 of Streptomyces fradiae also encodes three resistance genes (t/rB, t/rC and $t / r D)$ [15],

92 while in another example, Streptomyces toyacaensis, the vanHAX resistance cassette is

93 proximal to the vancomycin biosynthesis gene cluster, thereby encoding inherent

94 resistance [16].

95

96 Remote and pristine microbial communities provide a rich genetic resource to explore the

97 historical evolutionary origins of naturally occurring antibiotic resistance from the pre-

98 antibiotic era. Only in few pristine environments with limited anthropogenic influence (e.g.,

99 permafrost, glaciers, deep sea, and polar regions) can remnants of the above-described

100 ancient biological warfare mechanisms still be detected. These ARGs and resistant

101 bacteria evolving in pristine environments may therefore be considered the inherent

102 antibiotic resistance present in the environment [5].

104 We have recently reported the genomic and metabolic adaptations of epilithic biofilms to 105 windows of opportunities in glacier-fed streams (GFSs) [17]. For example, given the short

106 flow season during glacial melt, i.e. summer, the incentive to reproduce quickly while 107 conditions are favourable, is high. During these windows of opportunity, the necessity for 108 taxa to not only acquire physical niches, but also appropriate resources yields a 109 competitive environment. Within these biofilms, we observe complex cross-domain 
110 interactions between microorganisms to potentially mitigate the harsh nutrient and

111 environmental conditions of the GFSs. Additionally, owing to their complex biodiversity

112 [18] and generally oligotrophic conditions [19], epilithic biofilms are ideal model systems

113 for understanding BGCs and AMR. While oligotrophy may provide the basis for

114 competition over resources amongst microorganisms such as prokaryotes and (micro-

115 )eukaryotes. Our previous insights revealed that taxa such as Polaromonas,

116 Acidobacteria, and Methylotenera have strong interactions with eukaryotes such as algae

117 and fungi [17]. The inherent diversity allows for understanding the influence of AMR in

118 microbial interactions. For example, the accidental discovery of penicillin by Alexander

119 Fleming in 1928 based on bacterial-fungal interactions, [20], has since been expanded

120 upon by Netzker et al. [21]. They reported that microbial interactions lead to the production

121 of bioactive compounds including antibiotics that may shape the microbial consortia within

122 a community.

124 Here, to shed light on the role of AMR in shaping microbial communities within (relatively)

125 pristine environments, we used high-resolution metagenomics to investigate twenty-one

126 epilithic biofilms from glacier-fed streams. These samples were collected from 8 GFSs

127 spread across the Southern Alps in New Zealand and the Caucasus in Russia

128 (Supplementary Table 1). Herein, we found 29 categories of ARGs within the GFSs

129 across both bacterial and eukaryotic domains. Importantly, most of the AMR was found

130 in bacteria. We also identified antibacterial BGCs that were encoded both in bacterial and

131 eukaryotes suggesting extensive intra- and inter-domain competition. Our findings

132 demonstrate that microorganisms within biofilms from pristine environments not only

133 encode ARGs, but that they may potentially influence several features of epilithic biofilms 
134 such as biofilm formation, community assembly and/or maintenance, including conferring

135 mechanisms for competitive advantages under extreme conditions.

\section{Methods}

\section{Sampling and biomolecular extractions}

139 Eight GFSs were sampled in early- to mid-2019 from the New Zealand Southern Alps and

140 the Russian Caucasus, respectively, for a total of 21 epilithic biofilms (Supp. Table 1).

141 The biofilm samples were collected from each stream reach due to biofilms ranging from

142 abundant to absent, depending on stream geomorphology. One to three biofilm samples

143 were collected per reach (Supp. Table 1), taken using sterilized metal spatulas to scrape

144 rocks, followed by their immediate transfer to cryovials. Samples were immediately flash-

145 frozen in liquid nitrogen and stored at $-80^{\circ} \mathrm{C}$ until DNA was extracted. DNA from the

146 epilithic biofilms was extracted using a previously established protocol [22] adapted to a

147 smaller scale due to relatively high DNA concentrations. DNA quantification was

148 performed for all samples with the Qubit dsDNA HS kit (Invitrogen).

150 Sequencing and data processing for metagenomics

151 Random shotgun sequencing was performed on all epilithic biofilm DNA samples after

152 library preparation using the NEBNext Ultra II FS library kit. 50 ng of DNA was 153 enzymatically fragmented for $12.5 \mathrm{~min}$ and libraries were prepared with six PCR 154 amplification cycles. An average insert of $450 \mathrm{bp}$ was maintained for all libraries. Qubit 155 was used to quantify the libraries followed by sequencing at the Functional Genomics 156 Centre Zurich on a NovaSeq (Illumina) using a S4 flowcell. The metagenomic data was 
157 processed using the Integrated Meta-omic Pipeline (IMP v3.0; commit\# 9672c874

158 available at https://git-r3lab.uni.lu/IMP/imp3) [23]. IMP's workflow includes pre-

159 processing, contig assembly, genome reconstruction (metagenome-assembled

160 genomes, i.e. MAGs) and additional functional analysis of genes based on custom

161 databases in a reproducible manner [23].

162

Identification of antimicrobial resistance genes, antibiotic biosynthesis pathways and BGCs

165 For the prediction of ARGs the IMP-generated contigs were used as input for PathoFact

166 [24]. Identified ARGs were further collapsed into their respective AMR categories in

167 accordance with the Comprehensive Antibiotic Resistance Database (CARD) [25].

168 PathoFact uses an HMM-based search to identify homologous sequences across

169 genomic data, therefore possibly also detecting resistance genes within eukaryotic

170 genomic fragments. Subsequently, the raw read counts per ORF, obtained from

171 PathoFact, were determined using FeatureCounts [26].

173 To identify pathways for the biosynthesis of antibiotics, we assigned KEGG orthology

174 (KOs) identifiers to the ORFs using a hidden Markov model [27] (HMM) approach using

175 hmmsearch from HMMER 3.1 [28] with a minimum bit score of 40. Additionally, we linked

176 the identified KOs to their corresponding KEGG orthology pathways and extracted the

177 pathways annotated as antibiotic biosynthesis pathways by KEGG. Both the identified

178 ARGs and KEGG pathways were then further linked to associated bacterial taxonomies.

179 The bacterial and eukaryotic taxonomies were assigned using the PhyloDB and MMETSP

180 databases associated with EUKulele (commit\# fb8726a; available at 
181 https://github.com/AlexanderLabWHOI/EUKulele). Consensus taxonomy per contig was

182 then used for downstream analyses including association with ARGs.

184 We further identified BGCs within the MAGs using antiSMASH (ANTIbiotics \& Secondary 185 Metabolite Analysis SHell) [29] and annotated these using deepBGC [30]. To link BGCs 186 and ARGs, we linked the resistance genes to their associated assembled contigs, 187 followed by identifying the corresponding bins (MAGs) to which said contigs belonged.

\section{Data analysis}

190 The relative abundance of the ORFs was calculated based on the RNum_Gi method 191 described by Hu et al. [31]. Figures for the study, including visualizations derived from the 192 taxonomic and functional analyses, were created using version 3.6 of the $\mathrm{R}$ statistical 193 software package [32] and using the tidyverse package [33]. Alluvial plots were 194 generated using the ggalluvial package [34] while heatmaps were generated using the 195 ComplexHeatmap package [35] developed for R. The corresponding visualization and 196 analysis code is available at: https://gitr3lab.uni.lu/laura.denies/Rock_Biofilm_AMR.

\section{Results}

\section{Antimicrobial resistance in a pristine environment}

200 We characterised the resistomes of GFS epilithic biofilms and assessed the distribution 201 of AMR in twenty-one epilithic biofilm samples, across 8 individual glaciers originating 202 from the Southern Alps in New-Zealand (SA1, SA2, SA3 and SA4) and the Caucasus in 
203 Russia (CU1, CU2, CU3, CU4). In total, we identified a high number ( $\mathrm{n}=1840)$ of ARGs

204 within 29 categories of AMR, with similar AMR profiles observed across all GFSs (Fig.

205 1a, Supp. Fig. 1), except for SA2 and SA3 where the differences were driven by elevated

206 fluoroquinolone, glycopeptide and phenicol resistance, respectively. It is to be noted that

207 while ARGs refer to the genes encoding specific resistance, AMR categories derived from

208 metagenomic data in this context, typically reflect the functional potential associated with

209 respect to the resistance encoded. Of the identified AMR categories, beta-lactam and

210 multidrug resistance (i.e. resistance conferring protection against multiple antibiotic

211 classes), followed by aminoglycoside resistance, were found to be highly abundant in all

212 samples. We subsequently analysed the diversity of ARGs within the various resistance

213 categories and found beta-lactam resistance to represent the largest resistance category,

214 contributing 930 unique ARGs to the resistome. This was followed by multidrug (179 215 ARGs) and aminoglycoside (176 ARGs) resistance (Supp. Table 2). In contrast, some

216 resistance categories such as polymyxin and pleuromutilin resistance were only detected

217 at very low levels within the epilithic biofilm resistomes.

219 We further investigated the contribution of microbial populations to the resistome and 220 found contributions from both prokaryotes and eukaryotes (Fig. 1b). Prokaryotes within 221 this study refer to bacteria alone, since archaea encoded for an infinitesimal number of 222 ARGs (<0.000001\% RNum_Gl; Methods), and therefore were excluded from further

223 analyses. Among the eukaryotes, the phylum Ochrophyta (algae) was the dominant 224 contributor and encoded most of the AMR categories (Fig. 1c, Supp. Fig. 2a). In bacteria, 225 AMR was more evenly distributed with most of the phyla encoding ARGs across all 226 categories (Fig. 1c). However, members of the Alphaproteobacteria, Betaproteobacteria, 
227 and the Bacteroidetes/Chlorobi group encoded the highest overall ARG abundance (Fig.

228 1c, Supp. Fig. 2b). Additionally, AMR categories such as aminoglycoside, beta-lactam,

229 glycopeptide and rifamycin resistance (among others) were widely distributed in both

230 bacteria as well as among the eukaryotes. On the other hand, categories such as

231 aminocoumarin, bacitracin, and diaminopyrimidine resistance were found to be primarily

232 encoded by bacteria.

234 Antibiotic biosynthesis pathways and biosynthetic gene clusters

235 As described above, beta-lactam, multidrug and aminoglycoside resistance were the

236 most abundant resistance categories within GFS epilithic biofilms. This was not surprising

237 as beta-lactams and aminoglycosides are natural and prevalent compounds [36,37].

238 Furthermore, multidrug resistance is typically conferred via efflux machineries which were 239 also common in the GFS epilithic biofilms. These typically serve dual purposes in

240 particular for protein export within most bacteria [38]. Based on these results, it is

241 therefore highly likely that pristine environments such as GFSs potentially reflect the

242 spectrum of natural antibiotics and their resistance mechanisms, reinforcing their capacity

243 to serve as natural baselines for assessing enrichments and spread of AMR.

245 To further understand if these encoded resistance genes reflected natural antibiotic 246 pressure, we investigated pathways associated with antibiotic biosynthesis using the

247 KEGG database [39]. In total, we identified seven different pathways corresponding to 248 the biosynthesis of macrolides (MLS), ansamycins, glycopeptides (vancomycin), beta249 lactams (monobactam, penicillin and cephalosporin), aminoglycosides (streptomycin), 
250 and tetracyclines, which were present in various abundances in all samples (Supp. Fig.

251 3a). Importantly, the identified antibiotic synthesis genes thereby corresponded to the

252 resistance categories identified within the epilithic biofilms. Interestingly, in most of the

253 GFSs, antibiotic biosynthesis was primarily encoded by bacteria spanning multiple phyla

254 (Supp. Fig. 3b, Supp. Fig. 3c). Exceptions to these were GL11 and GL15 in which

255 biosynthesis pathways were equally distributed among eukaryotes, specifically

256 Ochrophyta, in addition to bacteria.

258 To further validate our observations, we assessed the abundance of BGCs, which are

259 known to encode genes for secondary metabolite synthesis, including antibiotics. We

260 found six different structural classes of BGCs by annotating 537 medium-to-high quality

261 (>50\% completion and $<10 \%$ contamination) bacterial and 30 eukaryotic MAGs using

262 antiSmash [29] and DeepBGC [30]. Using this ensemble approach we identified one or

263 more BGCs in most bacterial ( $n=490, \sim 91 \%$ of all bacterial MAGs) and eukaryotic $(n=28)$

264 MAGs. Of these BGCs, those annotated with an antibacterial function were dominant

265 across the microbial populations, represented here by the MAGs, and were found across

266 all phyla (Fig. 2a). Overall, a wider variety of BGCs associated with cytotoxic activity,

267 inhibitory, and antifungal mechanisms were also identified in bacteria. Eukaryotes, on the

268 other hand, encoded a high prevalence of antibacterial BGCs ( $\sim 93 \%$ of all eukaryotic

269 MAGs) (Fig. 2a). We further annotated those BGCs identified as antibacterial to

270 determine their subtypes and found that most of them were 'unknown' (Fig. 2b). However,

271 other identified subtypes include ribosomally synthesized and post-translationally

272 modified peptides (RiPPs) such as bacteriocins, along with NRPs, PKs, and terpenes. 
274 According to the resistance hypothesis [14], within or close to, each BGC there is at least

275 one gene conferring resistance to its encoded secondary metabolite. To test this, we

276 assessed whether the MAGs encoding a BGC also encoded corresponding ARGs. In line

277 with this hypothesis, we identified BGCs and their respective resistance genes in close

278 proximity to each other through their localization on the same contig. Consequently, we

279 identified various BGCs encoded together with ARGs in both the bacterial and eukaryotic

280 MAGs. For example, we found that an antibacterial BGC was encoded by Flavobacterium

281 spp. on the same contig as both MLS (macrolides, lincosamides and streptogramin) and

282 beta-lactam resistance genes (Fig. 2c). Incidentally, we also found that a candidate phyla

283 radiation (CPR) bacterium (Aalborg-AAW-1; phylum Patescibacteria) also encoded both

284 antibacterial BGC and MLS resistance on the same contig.

286 Discussion

287 Microbial reservoirs in pristine environments, with little to no impact from anthropogenic

288 selection pressures, provide the opportunity to investigate the natural propensity and

289 linked evolutionary origins of AMR. Here, by leveraging high-resolution metagenomics on

290 twenty-one epilithic biofilms, we assessed the resistomes of eight individual GFS epilithic 291 biofilms.

292

293 To date, while many studies have looked for novel antibiotics and resistance genes in 294 pristine environments such as the deep sea [40] or the polar regions [41], few have 295 explored the full diversity of antibiotic resistance in such environments [42,43]. Van 296 Goethem et al. [44] identified 117 naturally occurring ARGs associated with multidrug, 
297 aminoglycoside and beta-lactam resistance in pristine Antarctic soils. Similarly, D'Costa

298 et al. [4] identified a collection of ARGs encoding resistance to beta-lactams as well as

299 tetracyclines and glycopeptides in 30,000-year-old Beringian permafrost sediments. In

300 agreement with these previous studies, we identified 29 AMR categories, including the

301 previously mentioned resistance categories, in the studied biofilm communities. Among

302 these, the highest ARG abundance was associated with aminoglycoside and beta-lactam

303 resistance. Our study further suggests that although the overall abundance differs, the 304 epilithic resistome was highly similar in all GFSs, independent of origin (i.e. New Zealand

305 or Russia). Furthermore, our results agree with the results obtained in other resistomes

306 identified in pristine environments such as Antarctic soils and permafrost in terms of the

307 identified ARGs. Unlike previous studies, where ARGs were primarily associated with

308 bacteria, we report for the first time that AMR was associated with both bacteria and 309 eukaryotes in various abundances in environmental samples including GFSs. A previous

310 study by Brown et al. [45] reported that the IRS-HR (isoleucyl-tRNA synthetase - high

311 resistance) type gene conferring resistance against mupirocin was identified in

312 Staphylococcus aureus. More importantly, they suggested that horizontal gene transfer

313 led to the acquisition of IRS-HR genes by bacteria from eukaryotes [45]. Despite these

314 early reports, the contribution of eukaryotes to most resistomes, including from pristine

315 environments, has largely been unexplored thus far. An exception to this was the report

316 by Fairlamb et al. [46] who identified eukaryotic drug resistance, especially encoded by

317 fungi (Candida and Aspergillus) and parasites (Plasmodium and Trypanosoma).

318 However, most of these modes of resistance were highly specific towards particular drug

319 treatments [46]. Our results specifically revealed that taxa from the phylum Ochrophyta 
320 encoded resistance to 28 AMR categories and this was also reflected in other (micro-

321 )eukaryotes.

322

323 Apart from encoded resistance mechanisms, microalgae such as Ochrophyta have been

324 of interest as a source of (new) antimicrobial compounds $[47,48]$. In line with this, Martins

325 et al. suggested that extracts from different microalgae may potentially serve not only as

326 antimicrobial agents, but also as anti-cancer therapeutics. However, our present results

327 suggest that these taxa may also serve as environmental reservoirs for AMR itself. It is

328 however presently unclear whether this phenomenon confers advantages with respect to

329 niche occupation and protection against bacterial infection as well as whether the

330 eukaryotes are sensitive to the antibiotics produced by them.

332 Studies delving into the origins of AMR have reported that fecal pollution may explain ARG abundances in anthropogenically impacted environments [49]. This phenomenon

334 was also observed by Antelo et al. [50] and others [51] who detected ARGs in soils in Antarctica, especially in proximity to scientific bases. Although it is plausible that some of

336 the GFSs sampled in our study may indeed be under anthropogenic influence, in pristine

337 environments, AMR is most likely derived from natural antibiotics produced by

338 microorganisms as a competitive advantage. Microorganisms acquire resistance either 339 as a protective measure against other microorganisms [52,53] or as a self-defense 340 mechanism to prevent inadvertent suicide by damaging metabolites [14]. Accordingly, we

341 found both antibiotic biosynthesis pathways and BGCs within the epilithic resistomes. We 342 identified pathways for the biosynthesis of glycopeptides, beta-lactams, and 343 aminoglycosides, among others, concurrent with the high abundance of ARGs against 
344 said antibiotics. Additionally, we identified BGCs with a predicted antibacterial function in

345 both eukaryotes and bacteria. While a limited number of studies such as Waschulin et al.

346 [54] and Liao et al. [55], have shown BGCs in pristine environments, none of these studies

347 have contextualized the co-occurrence of BGCs with AMR. Hence, we not only found that

348 most of our MAGs contain BGCs, of which many have an antibacterial function, but also

349 found all MAGs to encode multiple resistance genes. Additionally, we found several BGCs

350 closely localized to ARGs on the same contig, thereby indicating an immediate self-

351 defense mechanism against the encoded secondary metabolites. This agrees with the

352 resistance hypothesis highlighted by Tran et al. stating that a gene conferring resistance

353 to potentially harmful metabolites produced by the organism are to be found within the

354 BGC-encoding operons [14]. We also observed that the recently identified CPR bacteria

355 [56] (in our case, phylum Patescibacteria) not only encoded for AMR but also harboured

356 genes associated with the production of molecules with antibacterial effects. Although

357 Patescibacteria have been identified in oligotrophic environments $[57,58]$ with carbon

358 and/or nutrient limitations similar to those observed for GFSs, it is plausible that their

359 ability to survive with minimal biosynthetic and metabolic pathways may indeed depend

360 on the expression of BGCs and AMR. At the time of writing, a preprint by Maatouk et al.

361 [59], described the presence of ARGs across publicly available CPR bacterial genomes.

362 In addition, we report the identification of AMR within GFS-derived CPR genomes, likely

363 as a means of competitive inhibition against other taxa. Alternatively, biofilms may also

364 allow for collective resistance, tolerance, and exposure protection to antibacterial

365 compounds [60]. The AMR and BGCs encoded by most phyla may therefore affect

366 cooperation and/or interactions associated with nutrient exchange, leading to the

367 privatization of public goods [60]. Such a phenomenon may be achieved due to the 
368 competition within taxa, both at the intra- and inter-species levels, via secretion of toxins

369 [53] and occupying spatial niches [61,62] thereafter. Furthermore, Stubbendieck and

370 Straight previously highlighted the multifaceted effects of bacterial competition which

371 include the potential taxation and subsequent increase in bacterial fitness [63]. Thus, the

372 in-situ competition within multi-species biofilms may allow for cross-phyla and cross-

373 domain interactions whilst simultaneously increasing the overall fitness of the

374 endogenous epilithic microbial community. Alternatively, these interactions or lack thereof

375 may shape the overall community including spatial organisation [64], especially in energy

376 limited systems such as the GFSs.

\section{Conclusions}

379 Epilithic biofilms are an integral and key mode of survival in extreme environments such 380 as glacier-fed stream ecosystems. Herein, we report that these biofilms provide critical 381 insights into the naturally occurring resistome. Our findings demonstrate that intra- and 382 inter-domain competition and survival mechanisms shed light on the ecological dimension 383 of microbial communities. Furthermore, we reveal the congruence of genes encoding for 384 both BGCs and AMR, in both bacteria and eukaryotes. More importantly, we highlight for 385 the first time the comprehensive AMR profile of CPR bacteria and of (micro-)eukaryotes. 386 Collectively, our results highlight underlying resistance mechanisms, including BGCs, 387 employed in 'biological warfare' in oligotrophic and challenging glacier-fed stream 388 ecosystems. 


\section{List of Abbreviations}

$391 \quad$ AMR: Antimicrobial resistance

392 ARGs: Antimicrobial resistance gene(s)

393 BGC: Biosynthetic gene clusters

394 CA: Caucasus

395 CPR: Candidate Phyla radiation

396 GFSs: Glacier-fed stream(s)

397 GL: Glacier

398 IRS-RS: isoleucyl-tRNA synthetase - high resistance

399 IMP: Integrate Meta-Omics Pipeline

400 KEGG: Kyoto Encyclopedia of Genes and Genomes

401 MAGs: Metagenome-assembled genome(s)

402 NRPS: Non-ribosomal peptide synthetases

403 PKS: Polyketide synthases (type I and type II)

404 RiPPs: Post-translationally modified peptide(s)

405 SA: Southern Alps

406

407 Declarations

408 Ethics approval and consent to participate

409 Not applicable

410 Consent for publication

411 Not applicable 


\section{Availability of data and material}

413 The Biosample accession IDs listed under Supp. Table 3 can be found on NCBI under

414 the BioProject accession\# PRJNA733707. The analyses code for IMP and downstream

415 analyses is detailed at https://git-r3lab.uni.lu/susheel.busi/nomis_pipeline. Binning and

416 manual refinement of eukaryotic MAGs was done as described here: https://git-

417 r3lab.uni.lu/susheel.busi/nomis_pipeline/-

418 /blob/master/workflow/notes/MiscEUKMAGs.md. All visualization and analysis code is

419 available at: https://git-r3lab.uni.lu/laura.denies/Rock_Biofilm_AMR.

\section{Competing interests}

421 The authors declare that they have no competing interests

\section{$422 \quad$ Funding}

423 This research has been supported by The NOMIS Foundation to TJB and the Swiss

424 National Science Foundation (CRSII5_180241) supporting SBB. LdN and PW are

425 supported by the Luxembourg National Research Fund (FNR; PRIDE17/11823097)

426 awarded to PW.

\section{Authors' contributions}

428 SBB, LdN, PW, and TJB conceived the project. PP extracted DNA, SBB and PP prepared

429 the metagenomic libraries for sequencing. SBB and LdN conceptualized and performed

430 the data analyses. SBB and LdN wrote the manuscript with PW and TJB, with significant

431 input and editing from all coauthors. 
Acknowledgements

433 We gratefully acknowledge the laboratory support from Emmy Marie Oppliger at EPFL

434 and Lea Grandmougin, Janine Habier, Laura Lebrun at the University of Luxembourg.

435 We also acknowledge the key input from Rashi Halder at the LCSB Sequencing Platform

436 regarding library preparation. We thank Patrick May and Cedric Christian Laczny for the

437 crucial insights into metagenomic processing. The computational analyses were

438 performed at the HPC facilities at the University of Luxembourg (https://hpc.uni.lu) [65].

440 Figure legends

441 Figure 1. Epilithic biofilms in GFSs harbour a diverse resistome

442 (a) Relative abundance of 29 AMR categories within 21 epilithic biofilms collected from

443 four New Zealand Southern Alps (SA) and four Russian Caucasus (CU) GFSs. (b) Bar

444 plots depicting the relative abundance of bacteria and eukaryotes encoding ARGs. (c)

445 Phylum-level representation of the AMR abundances across bacteria and eukaryotes.

446 Size of the closed circle indicates the normalised relative abundance (Rnum_Gi; see

447 Methods), whereby the color represents individual phyla.

449 Figure 2. Biosynthetic gene clusters indicate the resistome potential

450 (a) Heatmap depicting the overall abundance of BGCs identified across bacterial and

451 eukaryotic MAGs. The respective phyla are listed on the left while the coloured legend

452 represents the taxonomic order. (b) In-depth characterisation of the 'antibacterial' BGCs

453 found within all phyla and orders across medium-to-high quality MAGs. (c) Alluvial plots 
454 depicting the taxa where both BGCs and AMR were found adjacently on the same contig.

455 Colours indicate the genera associated with the MAGs.

457 Supplementary figure 1. Ordination analyses reveal the (dis)similarity of the GFS

458 resistomes

459 (a) Principal component analyses depicting the overall similarity of the individual GFS

460 resistomes. Each dot represents the resistome predicted from a single metagenome. SA:

461 Southern Alps. CU: Caucasus. (b) Biplot demonstrating the underlying factors, i.e. ARG

462 abundances across 29 AMR categories, driving the similarity within the GFS epilithic

463 resistomes.

464

465 Supplementary figure 2. Bacterial and eukaryotic phyla encode AMR

466 (a) Relative abundance of the bacteria associated with AMR. The stacked bar plots are

467 facetted by the individual GFSs where the epilithic biofilms were collected. The colors

468 represent the individual phyla. (b) Stacked bar plots indicating the relative abundance of 469 the AMR encoded by eukaryotes.

471 Supplementary figure 3. Antibiotic synthesis pathway assessment via KEGG

472 orthology

473 (a) Relative abundance of KEGG pathways associated with antibiotic synthesis across

474 the 21 epilithic biofilms. (b) Bar plots indicating the relative abundance of the antibiotic

475 associated KEGG pathways mediated by bacteria and eukaryotes. (c) Normalised relative

476 abundance of pathways associated with antibiotic production in the KEGG database, 477 juxtaposed with the various phyla encoding these genes. 
479 Supplementary data

480 Supplementary table 1. Sample metadata

481 Supplementary table 2. List of ARGs identified across 21 GFS epilithic biofilms

482 Supplementary table 3. NCBI accession metadata

483

484 References

485 1. Stanton IC, Bethel A, Leonard AFC, Gaze WH, Garside R. What is the research evidence 486 for antibiotic resistance exposure and transmission to humans from the environment? A $487 \quad$ systematic map protocol. Environ Evid. 2020;9: 12.

488 2. Balasegaram M. Learning from COVID-19 to Tackle Antibiotic Resistance. ACS Infect Dis. $489 \quad 2021 ; 7: 693-694$.

490 3. Wright GD. The antibiotic resistome: the nexus of chemical and genetic diversity. Nat Rev $491 \quad$ Microbiol. 2007;5: 175-186.

492 4. D'Costa VM, King CE, Kalan L, Morar M, Sung WWL, Schwarz C, et al. Antibiotic 493 resistance is ancient. Nature. 2011;477: 457-461.

494 5. Scott LC, Lee N, Aw TG. Antibiotic Resistance in Minimally Human-Impacted

495 Environments. Int J Environ Res Public Health. 2020;17. doi:10.3390/ijerph17113939

496 6. Tyc O, Song C, Dickschat JS, Vos M, Garbeva P. The Ecological Role of Volatile and 497 Soluble Secondary Metabolites Produced by Soil Bacteria. Trends Microbiol. 2017;25: 280$498 \quad 292$.

499 7. Chen R, Wong HL, Kindler GS, MacLeod FI, Benaud N, Ferrari BC, et al. Discovery of an 
Abundance of Biosynthetic Gene Clusters in Shark Bay Microbial Mats. Front Microbiol. 2020;11: 1950.

502

8. Demain AL, Fang A. The Natural Functions of Secondary Metabolites. In: Fiechter A, editor. History of Modern Biotechnology I. Berlin, Heidelberg: Springer Berlin Heidelberg; 2000. pp. $1-39$.

9. Newman DJ, Cragg GM. Natural Products as Sources of New Drugs from 1981 to 2014. J Nat Prod. 2016;79: 629-661.

10. Medema $\mathrm{MH}$, Kottmann R, Yilmaz $\mathrm{P}$, Cummings $\mathrm{M}$, Biggins JB, Blin $\mathrm{K}$, et al. Minimum Information about a Biosynthetic Gene cluster. Nat Chem Biol. 2015;11: 625-631.

11. Martinet L, Naômé A, Deflandre B, Maciejewska M, Tellatin D, Tenconi E, et al. A Single Biosynthetic Gene Cluster Is Responsible for the Production of Bagremycin Antibiotics and Ferroverdin Iron Chelators. MBio. 2019;10. doi:10.1128/mBio.01230-19

12. Martínez-Núñez MA, López VEL y. Nonribosomal peptides synthetases and their applications in industry. Sustainable Chemical Processes. 2016;4: 1-8.

514 13. Ridley CP, Lee HY, Khosla C. Evolution of polyketide synthases in bacteria. Proc Natl Acad Sci U S A. 2008;105: 4595-4600.

14. Tran PN, Yen M-R, Chiang C-Y, Lin H-C, Chen P-Y. Detecting and prioritizing biosynthetic gene clusters for bioactive compounds in bacteria and fungi. Appl Microbiol Biotechnol.

15. Cundliffe E, Bate N, Butler A, Fish S, Gandecha A, Merson-Davies L. The tylosinbiosynthetic genes of Streptomyces fradiae. Antonie Van Leeuwenhoek. 2001;79: 229-234.

16. Kwun MJ, Hong H-J. Genome Sequence of Streptomyces toyocaensis NRRL 15009, Producer of the Glycopeptide Antibiotic A47934. Genome Announc. 2014;2. 
doi:10.1128/genomeA.00749-14

524 17. Busi SB, Bourquin M, Fodelianakis S, Michoud G, Kohler TJ, Peter H, et al. Genomic and

525 metabolic adaptations of biofilms to ecological windows of opportunities in glacier-fed streams. bioRxiv. 2021. p. 2021.10.07.463499. doi:10.1101/2021.10.07.463499

18. Battin TJ, Besemer K, Bengtsson MM, Romani AM, Packmann Al. The ecology and biogeochemistry of stream biofilms. Nat Rev Microbiol. 2016;14: 251-263.

19. Battin TJ, Wille A, Sattler B, Psenner R. Phylogenetic and functional heterogeneity of sediment biofilms along environmental gradients in a glacial stream. Appl Environ Microbiol.

20. Gaynes R. The Discovery of Penicillin-New Insights After More Than 75 Years of Clinical Use. Emerg Infect Dis. 2017;23: 849. interactions trigger the production of antibiotics. Curr Opin Microbiol. 2018;45: 117-123. biomolecular extraction for metagenomic analysis of microbial biofilms from high-mountain streams. PeerJ. 2020;8: e9973.

23. Narayanasamy S, Jarosz Y, Muller EEL, Heintz-Buschart A, Herold M, Kaysen A, et al. IMP: a pipeline for reproducible reference-independent integrated metagenomic and pipeline for the prediction of virulence factors and antimicrobial resistance genes in metagenomic data. Microbiome. 2021;9: 49. 
2020: antibiotic resistome surveillance with the comprehensive antibiotic resistance database. Nucleic Acids Res. 2020;48: D517-D525.

26. Liao Y, Smyth GK, Shi W. featureCounts: An efficient general-purpose program for assigning sequence reads to genomic features. arXiv [q-bio.GN]. 2013. Available:

27. Yoon B-J. Hidden Markov Models and their Applications in Biological Sequence Analysis. http://arxiv.org/abs/1305.3347

28. Eddy SR. Accelerated Profile HMM Searches. PLoS Comput Biol. 2011;7: e1002195.

29. Blin K, Shaw S, Kloosterman AM, Charlop-Powers Z, van Wezel GP, Medema MH, et al. antiSMASH 6.0: improving cluster detection and comparison capabilities. Nucleic Acids Res. 2021;49: W29-W35.

30. Hannigan GD, Prihoda D, Palicka A, Soukup J, Klempir O, Rampula L, et al. A deep learning genome-mining strategy for biosynthetic gene cluster prediction. Nucleic Acids

31. Hu Y, Yang X, Qin J, Lu N, Cheng G, Wu N, et al. Metagenome-wide analysis of antibiotic resistance genes in a large cohort of human gut microbiota. Nat Commun. 2013;4: 2151.

32. Computing R, Others. R: A language and environment for statistical computing. Vienna: $R$ Core Team. 2013. Available: https://www.yumpu.com/en/document/view/6853895/r-alanguage-and-environment-for-statistical-computing

33. Wickham H, Averick M, Bryan J, Chang W, McGowan L, François R, et al. Welcome to the tidyverse. J Open Source Softw. 2019;4: 1686. 2017. 
35. Gu Z, Eils R, Schlesner M. Complex heatmaps reveal patterns and correlations in multidimensional genomic data. Bioinformatics. 2016;32: 2847-2849.

571

572

573

574

36. Krause KM, Serio AW, Kane TR, Connolly LE. Aminoglycosides: An Overview. Cold Spring Harb Perspect Med. 2016;6. doi:10.1101/cshperspect.a027029

37. Tahlan K, Jensen SE. Origins of the $\beta$-lactam rings in natural products. J Antibiot . 2013;66: 401-410.

38. Borges-Walmsley MI, McKeegan KS, Walmsley AR. Structure and function of efflux pumps that confer resistance to drugs. Biochem J. 2003;376: 313-338.

39. Kanehisa M, Goto S. KEGG: Kyoto Encyclopedia of Genes and Genomes. Nucleic Acids Res. 2000;28: 27-30.

40. Tortorella E, Tedesco P, Palma Esposito F, January GG, Fani R, Jaspars M, et al. Antibiotics from Deep-Sea Microorganisms: Current Discoveries and Perspectives. Mar Drugs. 2018;16. doi:10.3390/md16100355

41. McCann CM, Christgen B, Roberts JA, Su J-Q, Arnold KE, Gray ND, et al. Understanding drivers of antibiotic resistance genes in High Arctic soil ecosystems. Environ Int. 2019;125: 497-504.

42. Yuan K, Yu K, Yang R, Zhang Q, Yang Y, Chen E, et al. Metagenomic characterization of antibiotic resistance genes in Antarctic soils. Ecotoxicol Environ Saf. 2019;176: 300-308.

43. Centurion VB, Delforno TP, Lacerda-Júnior GV, Duarte AWF, Silva LJ, Bellini GB, et al. Unveiling resistome profiles in the sediments of an Antarctic volcanic island. Environ Pollut. 2019;255: 113240.

44. Van Goethem MW, Pierneef R, Bezuidt OKI, Van De Peer Y, Cowan DA, Makhalanyane TP. A reservoir of "historical" antibiotic resistance genes in remote pristine Antarctic soils. 
Microbiome. 2018;6: 40.

45. Brown JR, Zhang J, Hodgson JE. A bacterial antibiotic resistance gene with eukaryotic origins. Curr Biol. 1998;8: R365-7.

46. Fairlamb AH, Gow NAR, Matthews KR, Waters AP. Drug resistance in eukaryotic microorganisms. Nat Microbiol. 2016;1: 16092.

47. Silva A, Silva SA, Carpena M, Garcia-Oliveira P, Gullón P, Barroso MF, et al. Macroalgae as a Source of Valuable Antimicrobial Compounds: Extraction and Applications. Antibiotics

48. Martins RM, Nedel F, Guimarães VBS, da Silva AF, Colepicolo P, de Pereira CMP, et al. Macroalgae Extracts From Antarctica Have Antimicrobial and Anticancer Potential. Front

49. Karkman A, Pärnänen K, Larsson DGJ. Fecal pollution can explain antibiotic resistance gene abundances in anthropogenically impacted environments. Nat Commun. 2019;10: 80.

50. Antelo V, Giménez M, Azziz G, Valdespino-Castillo P, Falcón LI, Ruberto LAM, et al. Metagenomic strategies identify diverse integron-integrase and antibiotic resistance genes in the Antarctic environment. Microbiologyopen. 2021;10. doi:10.1002/mbo3.1219

51. Hernández F, Calısto-Ulloa N, Gómez-Fuentes C, Gómez M, Ferrer J, González-Rocha G, et al. Occurrence of antibiotics and bacterial resistance in wastewater and sea water from the Antarctic. J Hazard Mater. 2019;363: 447-456.

52. Reygaert WC. An overview of the antimicrobial resistance mechanisms of bacteria. AIMS Microbiol. 2018;4: 482-501.

613 53. Granato ET, Meiller-Legrand TA, Foster KR. The Evolution and Ecology of Bacterial Warfare. Curr Biol. 2019;29: R521-R537. 
615 54. Waschulin V, Borsetto C, James R, Newsham KK, Donadio S, Corre C, et al. Biosynthetic

616 potential of uncultured Antarctic soil bacteria revealed through long-read metagenomic

617 sequencing. ISME J. 2021. doi:10.1038/s41396-021-01052-3

618 55. Liao L, Su S, Zhao B, Fan C, Zhang J, Li H, et al. Biosynthetic Potential of a Novel Antarctic

619 Actinobacterium Marisediminicola antarctica ZS314T Revealed by Genomic Data Mining

620 and Pigment Characterization. Mar Drugs. 2019;17. doi:10.3390/md17070388

621 56. Hug LA, Baker BJ, Anantharaman K, Brown CT, Probst AJ, Castelle CJ, et al. A new view 622 of the tree of life. Nat Microbiol. 2016;1: 16048.

623 57. Tian R, Ning D, He Z, Zhang P, Spencer SJ, Gao S, et al. Small and mighty: adaptation of 624 superphylum Patescibacteria to groundwater environment drives their genome simplicity.

$625 \quad$ Microbiome. 2020;8: 51.

58. Vigneron A, Cruaud P, Langlois V, Lovejoy C, Culley Al, Vincent WF. Ultra-small and abundant: Candidate phyla radiation bacteria are potential catalysts of carbon transformation in a thermokarst lake ecosystem. Limnol Oceanogr Lett. 2020;5: 212-220.

59. Maatouk M, Ibrahim A, Rolain J-M, Merhej V, Bittar F. Small and equipped: the rich repertoire of antibiotic resistance genes in Candidate Phyla Radiation genomes. bioRxiv.

60. Bottery MJ, Pitchford JW, Friman V-P. Ecology and evolution of antimicrobial resistance in 2021. p. 2021.07.02.450847. doi:10.1101/2021.07.02.450847

634 61. Bottery MJ, Passaris I, Dytham C, Wood AJ, van der Woude MW. Spatial Organization of Expanding Bacterial Colonies Is Affected by Contact-Dependent Growth Inhibition. Curr Biol. 2019;29: 3622-3634.e5. 
$638 \quad$ competition. ISME J. 2015;9: 139-149.

639 63. Stubbendieck RM, Straight PD. Multifaceted Interfaces of Bacterial Competition. J

$640 \quad$ Bacteriol. 2016;198: 2145-2155.

641 64. Estrela S, Brown SP. Community interactions and spatial structure shape selection on

$642 \quad$ antibiotic resistant lineages. PLoS Comput Biol. 2018;14: e1006179.

643 65. Varrette S, Bouvry P, Cartiaux H, Georgatos F. Management of an academic HPC cluster:

644 The UL experience. 2014 International Conference on High Performance Computing

645 Simulation (HPCS). 2014. pp. 959-967. 


\section{Figure 1}

a
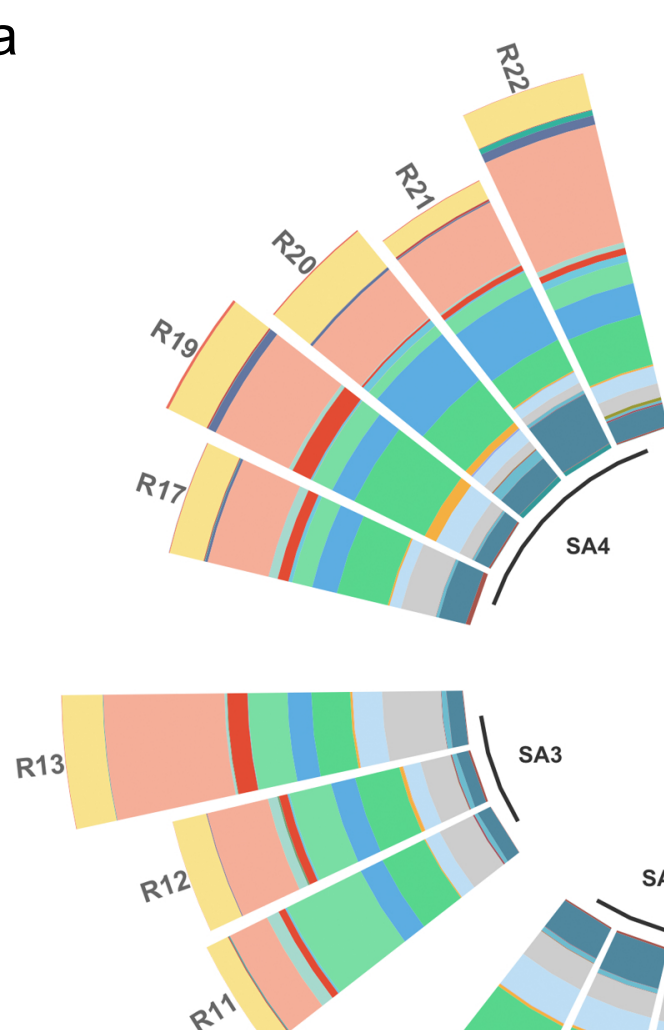

\section{.}
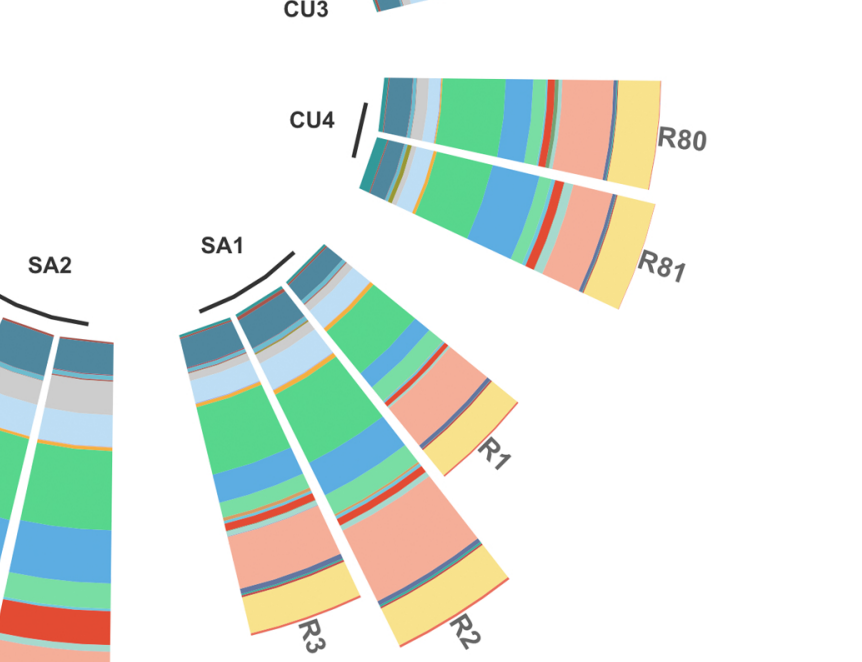

\section{AMR category}

\begin{tabular}{|l}
\hline acridine_dye \\
\hline aminocoumarin \\
aminoglycoside \\
\hline aminoglycoside:aminocoumarin \\
\hline antibacterial_free_fatty_acids \\
\hline bacitracin \\
\hline beta-lactam \\
\hline bicyclomycin \\
\hline diaminopyrimidine \\
\hline elfamycin \\
\hline fluoroquinolone \\
fosfomycin \\
fusidic-acid \\
\hline glycopeptide \\
\hline MLS
\end{tabular}

\begin{tabular}{|l|l|}
\hline multidrug \\
\hline mupirocin \\
\hline nitroimidazole \\
\hline nucleoside \\
peptide \\
\hline phenicol \\
\hline pleuromutilin \\
\hline polyamine:peptide \\
\hline polymyxin \\
\hline rifamycin \\
\hline sulfonamide \\
tetracycline \\
\hline triclosan \\
Unclassified
\end{tabular}

bioRxiv preprint doi: https://doi.org/10.1 101/2021.11.18.469141; this version posted November 19, 2021. The copyright holder for this preprint (which was not certified by peer review) is the author/funder, who has granted bioRxiv a license to display the preprint in perpetuity. It is made

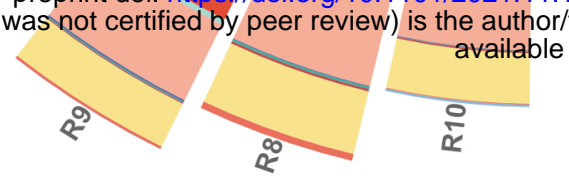

b

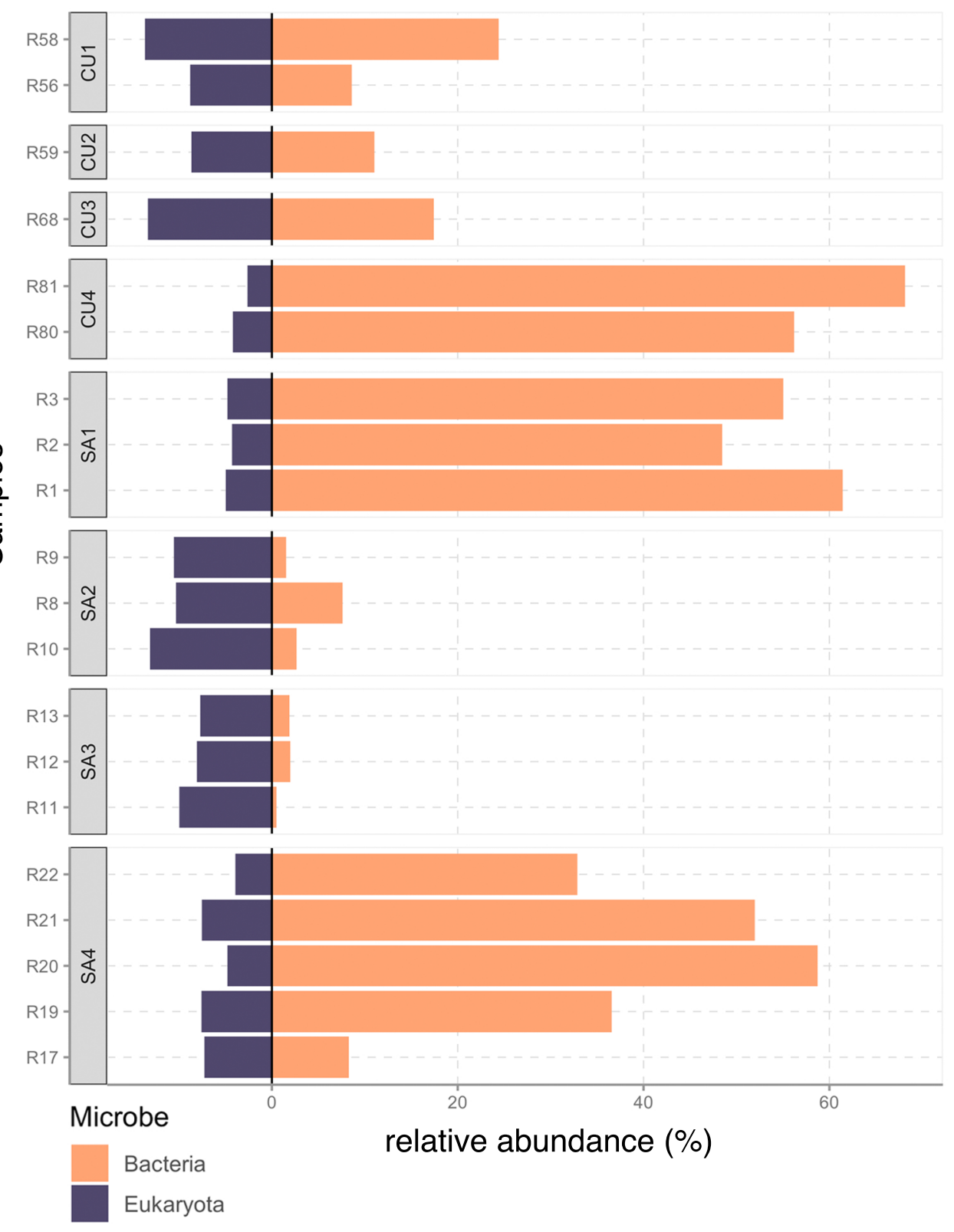

C

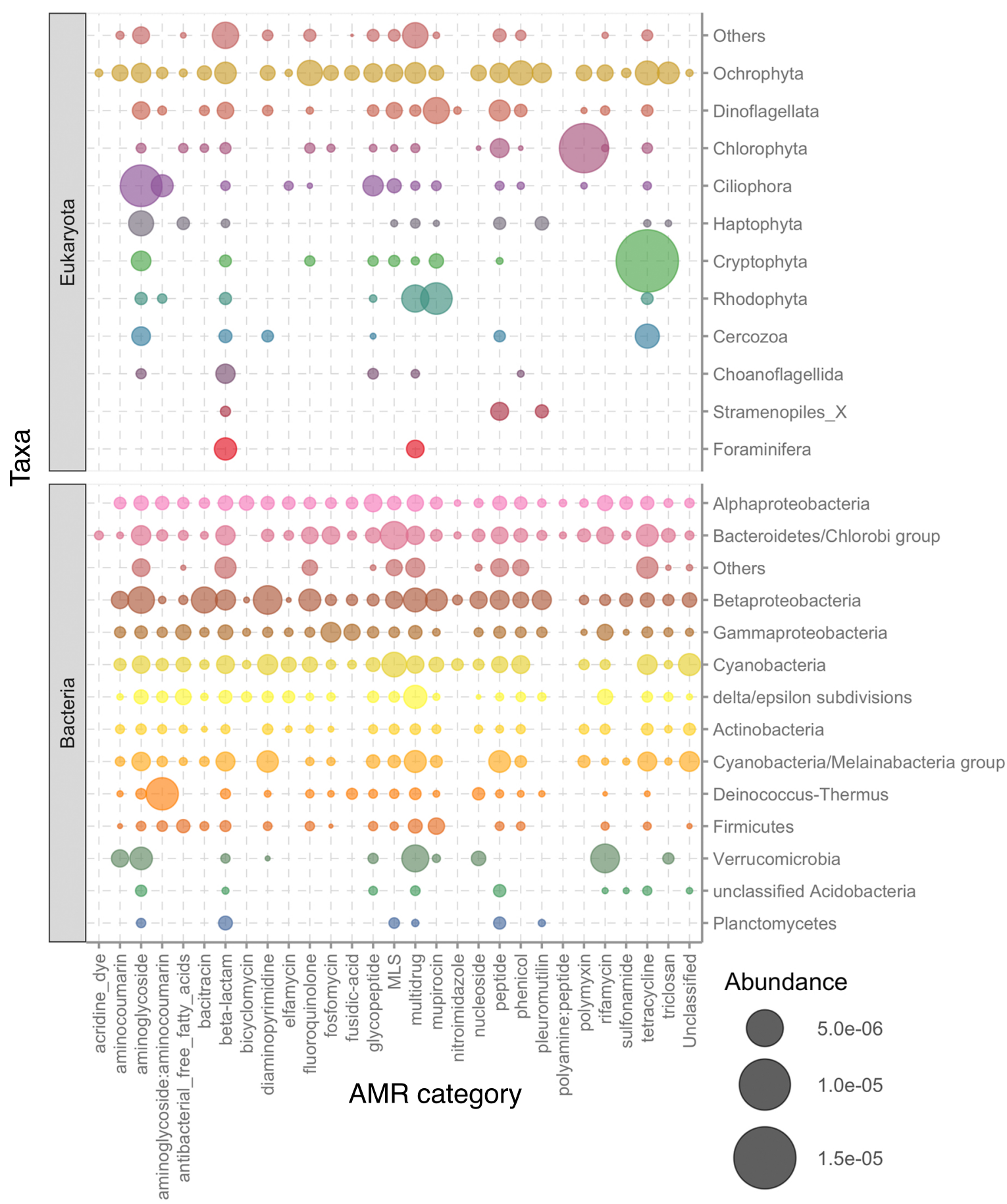




\section{Figure 2}

\section{a}

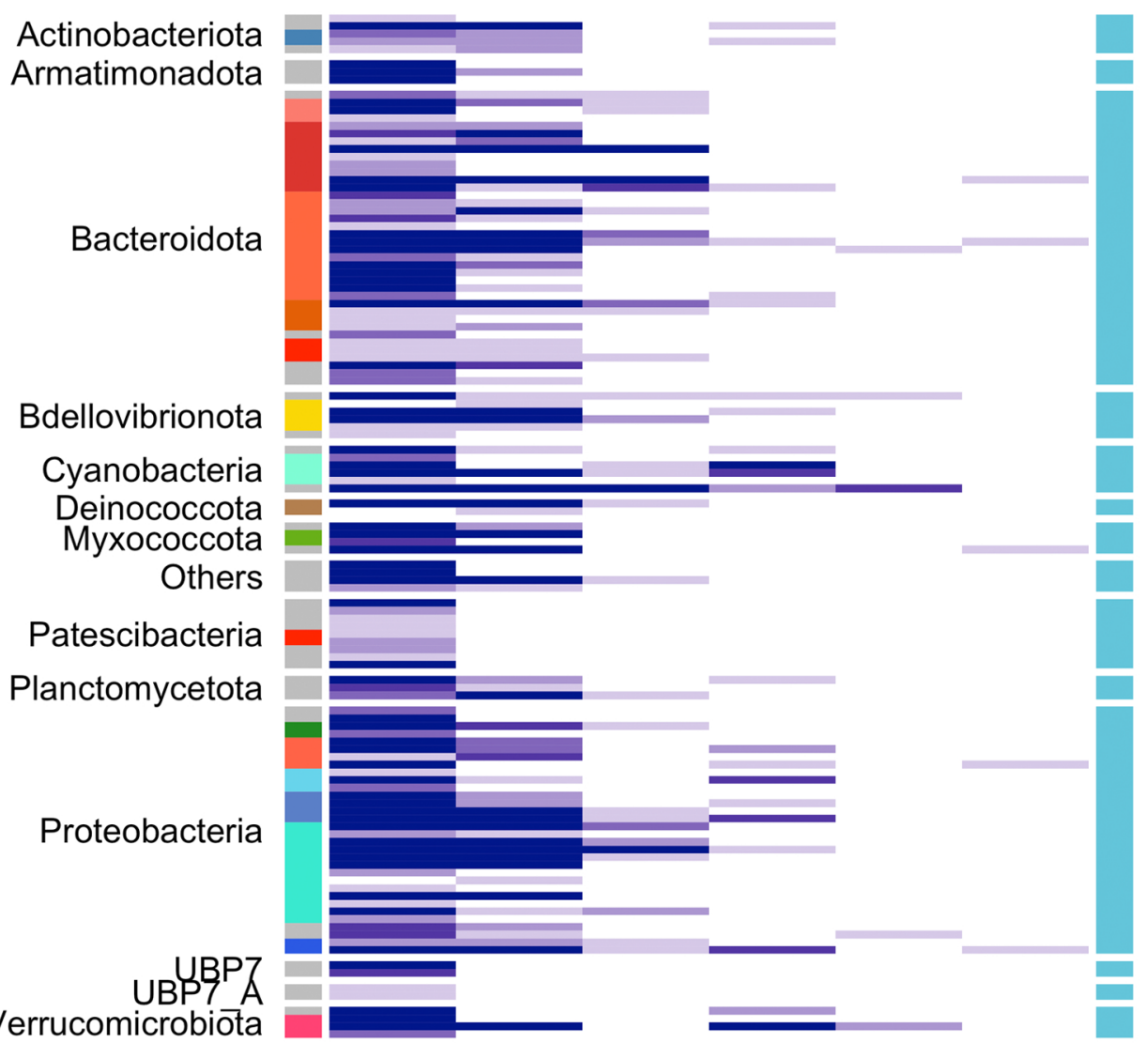

b

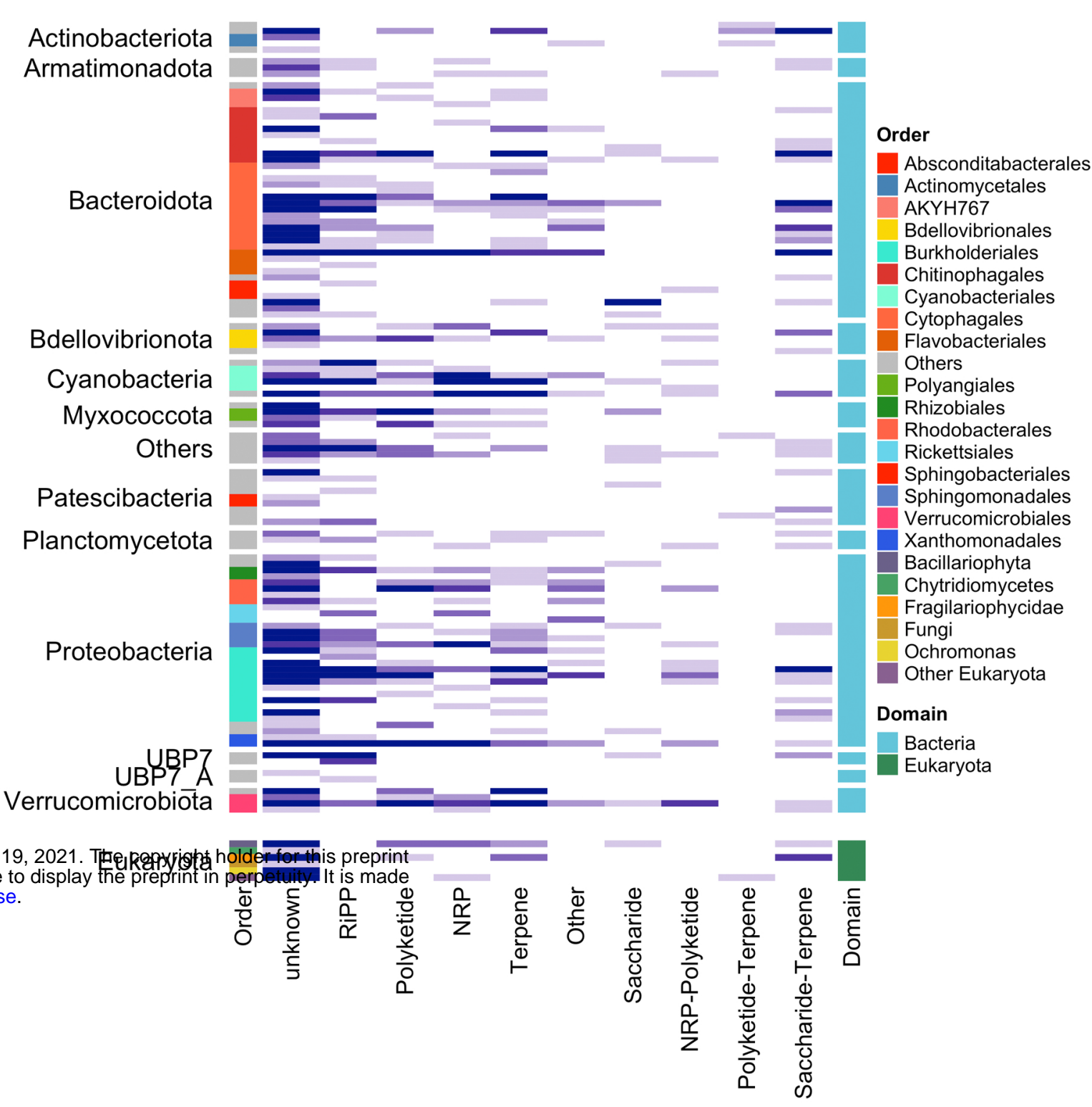

C

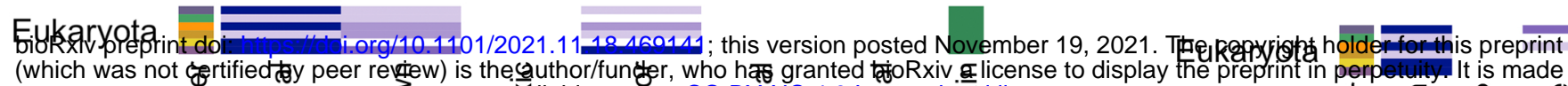

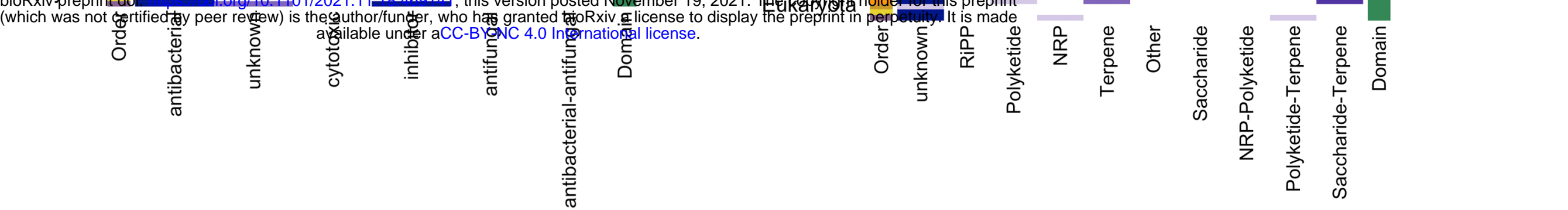

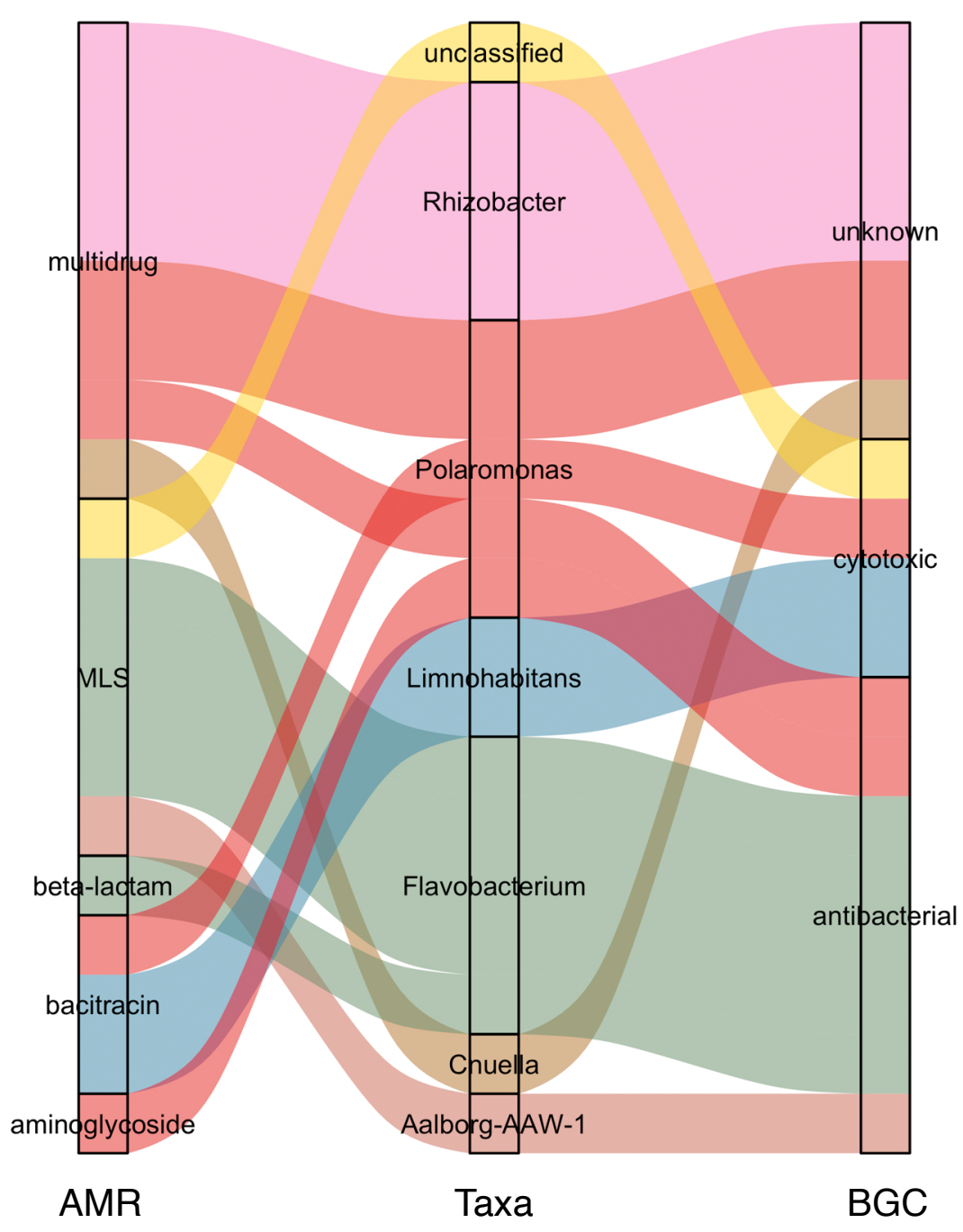




\section{Supplementary Figure 1}

a
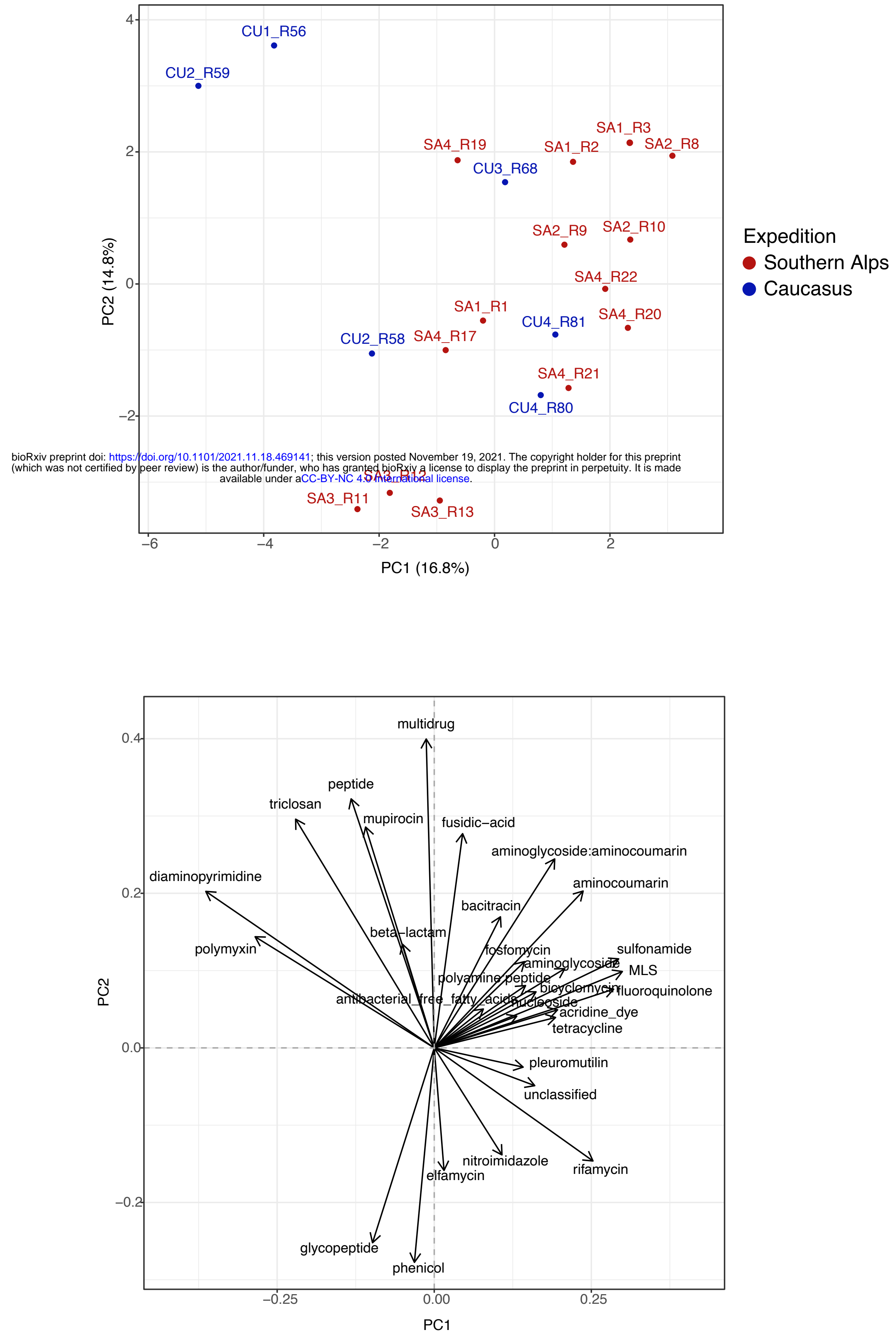
Supplementary Figure 2

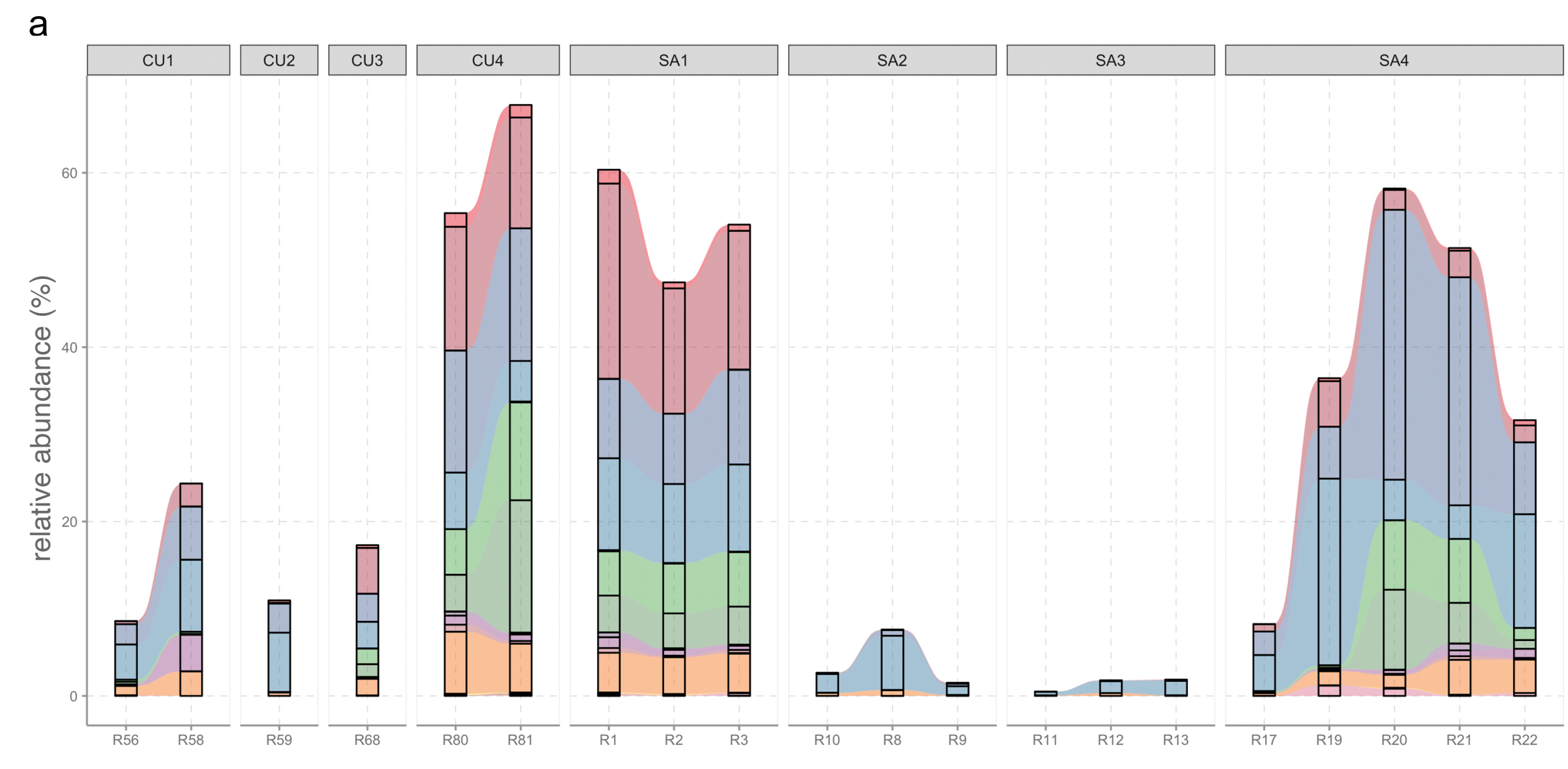

Bacteria

$\square$ Actinobacteria

Alphaproteobacteria

Aquificae

Bacteroidetes/Chlorobi group

Betaproteobacteria

Calditrichae

Chloroflexi

Cyanobacteria

Cyanobacteria/Melainabacteria group

Deferribacteres

Deinococcus-Thermus

delta/epsilon subdivision

Firmicutes

Fusobacteriia

Gammaproteobacteria

Gammaproteob

Oligoflexia

Planctomycetes

Spirochaetia

Synergistia

Tenericutes

Thermodesulfobacteria

Thermotogae

unclassified Acidobacteria

unclassified Bacteria (miscellaneous)

Verrucomicrobia

Zetaproteobacteria

b bioRxiv preprint doi: https://doi.org/10.1101/2021.11.18.469141; this version posted November 19, 2021. The copyright holder for this preprint

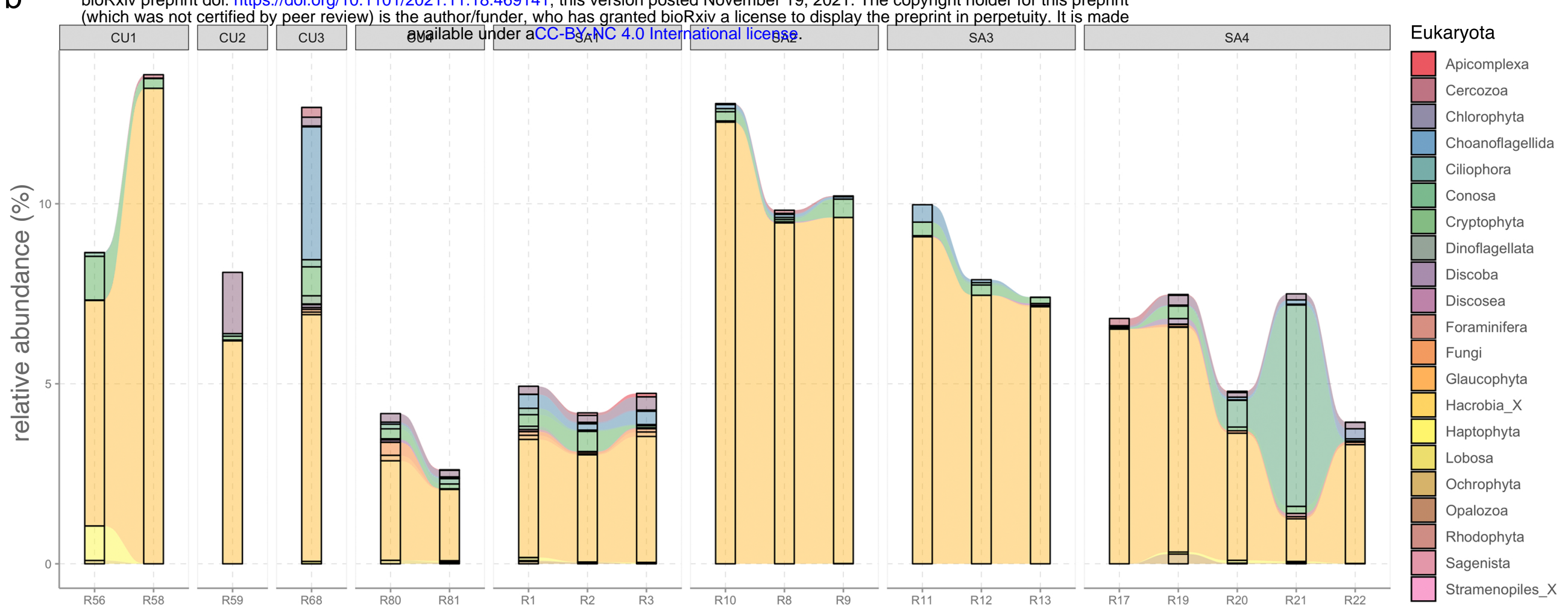




\section{Supplementary Figure 3}

a
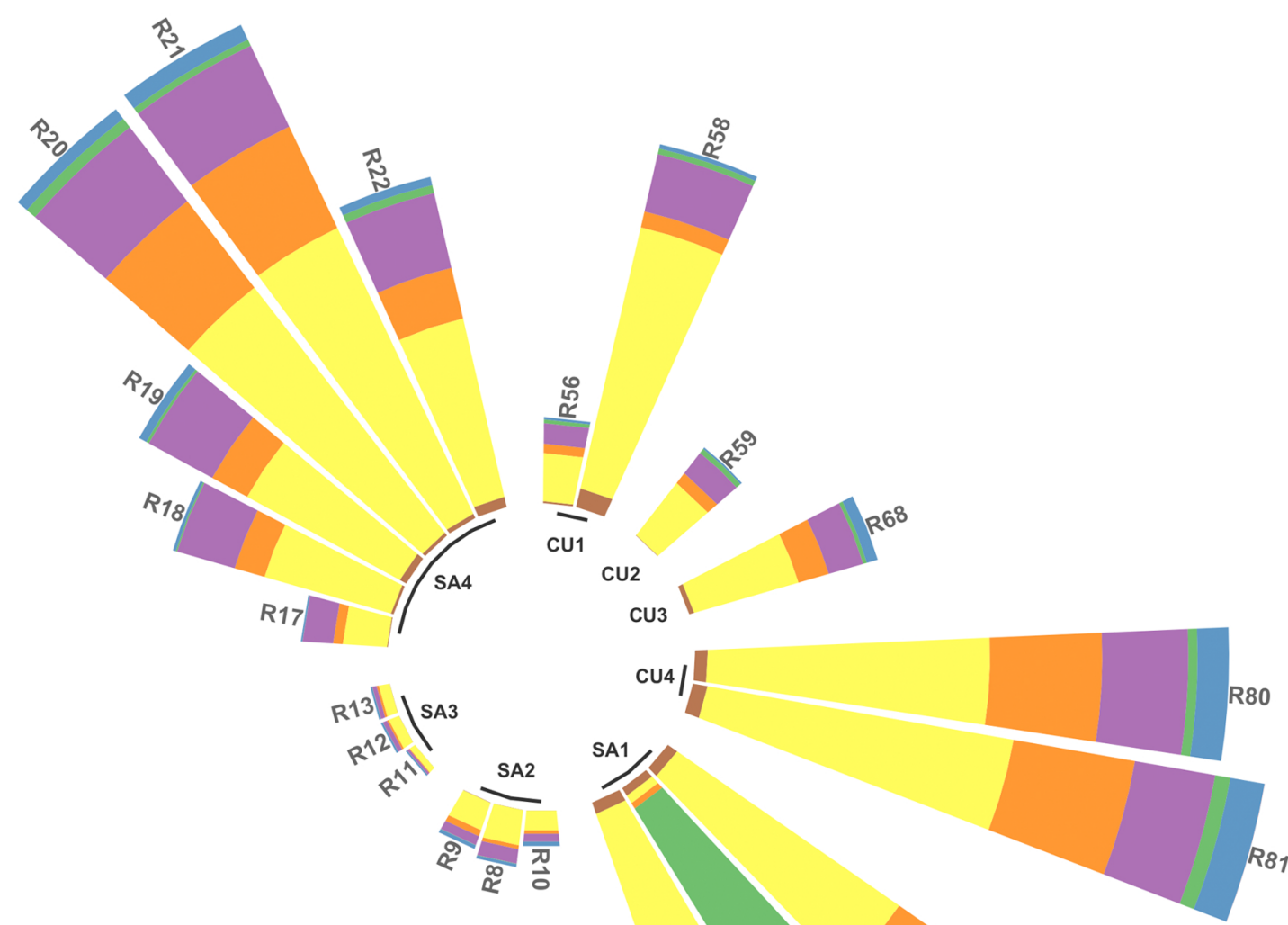

KEGG pathway

Biosynthesis of 12-, 14- and 16-membered macrolides Biosynthesis of ansamycins

Biosynthesis of vancomycin group antibiotics

Monobactam biosynthesis

bioRxiv preprint doi: https://doi.org/10.1101/2021.11.18.469141; this version posted November 19, 2021. The copyright holder for this preplfinenicillin and cephalosporin biosynthesis

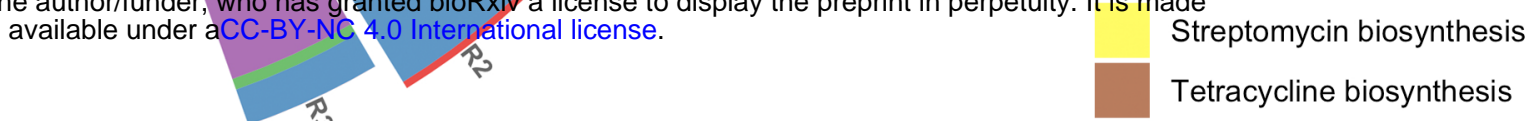

b

C
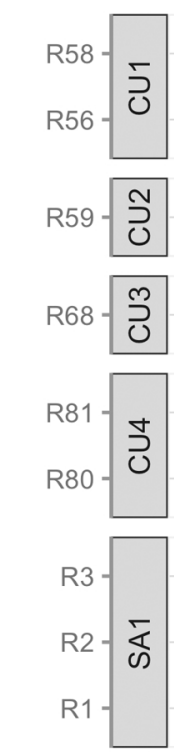

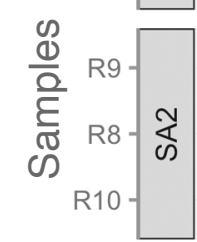

R12-

$\mathrm{R} 22-$
$\mathrm{R} 21-$
$\mathrm{R} 20-$
$\mathrm{R} 19$
$\mathrm{R} 18-$
$\mathrm{R} 17-$

\section{Microbe}

Bacteria

Eukaryota
Tetracycline biosynthesis

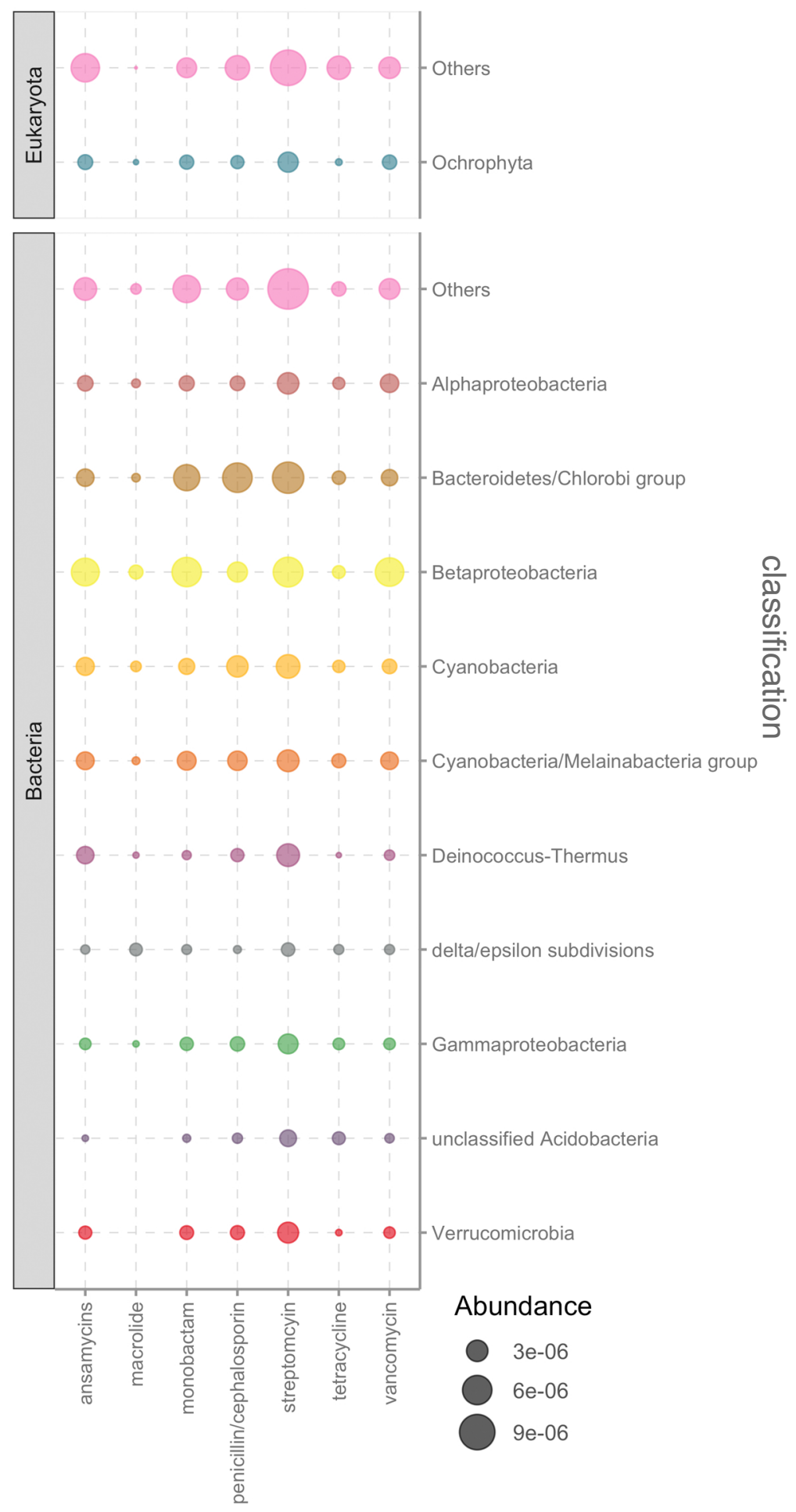

KEGG pathway 